\title{
NATURAL CUBIC ELEMENT FORMULATION AND INFINITE DOMAIN MODELLING FOR POTENTIAL FLOW PROBLEMS
}

\author{
G. A. MOHR ${ }^{1}$ and A. S. POWER ${ }^{1}$
}

(Received 31 October, 2000; revised 10 January, 2002)

\begin{abstract}
A simple formulation of a $9 \mathrm{df}$ cubic Hermitian finite element for potential flow problems is given, using the interpolation of the $\mathrm{BCIZ}$ element and after Argyris, defining natural velocities parallel to the element sides. Consistent loads for body forces are also derived and it is shown that these are necessary to obtain accurate results when body forces are significant. Example problems include those of infinite domains for which simple conditions at infinity are used.
\end{abstract}

\section{Introduction}

Finite elements for harmonic problems governed by Laplace's equation $\nabla^{2} \phi=0$ and pseudo-harmonic problems governed by $\nabla^{2} \phi=$ constant have very wide application. Examples are plane torsion, potential flow and electromagnetic fields [15, 18]. For these problems Lagrangian triangular elements with three or six nodes (and df) are commonly used $[5,15]$. In the present work, however, a cubic Hermitian element with freedoms $\phi, \partial \phi / \partial x, \partial \phi / \partial y$ at its vertices is used.

First the element of Mohr and Mohr [13] was used. The 9 global freedoms are transformed $[11,13]$ to the values of $\phi$ at the vertices and the third points on each side. By employing a simple approximation for $\phi$ at the centroid (in terms of the other nine values [13]) a complete Lagrangian cubic interpolation (in areal coordinates) is able to be used. Element formulation is straightforward.

Subsequently, however, it was found that the BCIZ element [3] (appropriately modified for potential problems) gave identical results for all the problems under study. It was then shown that the interpolations of the Mohr and Mohr and BCIZ elements are exactly equivalent [10].

\footnotetext{
'International Arts and Sciences College, 68 Tulip Grove, Cheltenham VIC 3192, Australia.

(C) Australian Mathematical Society 2003, Serial-fee code 1446-181 1/03
} 


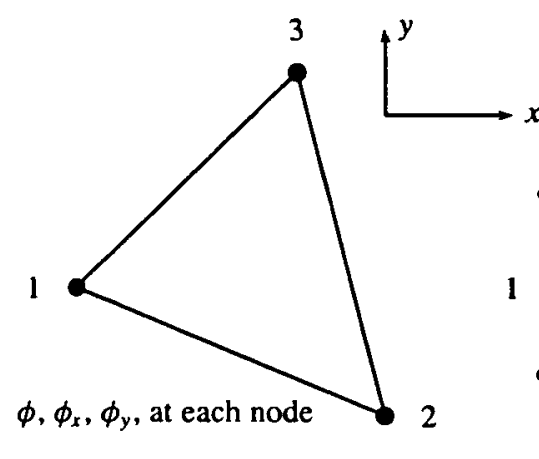

(a)

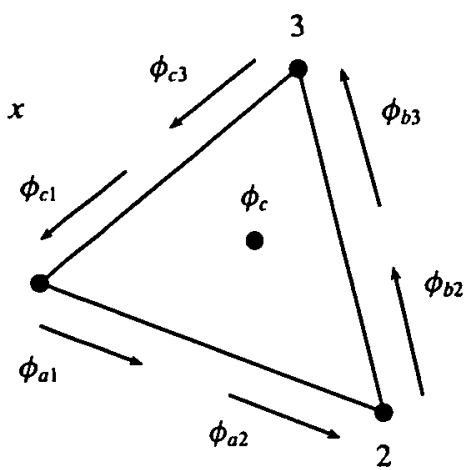

(b)

FigURE 1. (a) 9 df global element, (b) $10 \mathrm{df}$ local element.

The interpolation functions of the BCIZ element were originally obtained by somewhat intuitive arguments [18], but are derived from first principles in the present paper.

As the $\mathrm{BCIZ}$ element is, perhaps, the simplest reasonably accurate $9 \mathrm{df}$ Hermitian triangle, a simple formulation for this is given in the present work, complete with simple explicit formulae for the consistent loads for body forces. Of note too is that local natural velocities parallel to the element sides are defined in like fashion to the natural strains of Argyris [2].

The element is tested on a simple rectilinear flow problem. Then to test its convergence it is applied to the plane torsion problem. It is found that, provided that consistent loads are used to approximate body forces, the element is much more accurate than the popular six-node triangle of Argyris [1].

Next the element is applied to the classical problem of flow around a cylinder [7], yielding much better results than 3 and $6 \mathrm{df}$ Lagrangian elements.

Finally the problem of a point source/sink in an infinite domain is considered using both the $6 \mathrm{df}$ and $9 \mathrm{df}$ elements and simple conditions at infinity similar to the 'elastic boundary conditions' used by Mohr and Power [14] for plane thermoelasticity problems. With the use of these, only a few elements are needed to approximately model such problems.

\section{Element formulation}

Figure 1 (a) shows the global freedoms for the element, namely the potential $\phi$ and its Cartesian derivatives $\phi_{x}=\partial \phi / \partial x, \phi_{y}=\partial \phi / \partial y$ at each node. 
Defining natural derivatives

$$
\phi_{a}=\partial \phi / \partial s_{a}, \quad \phi_{b}=\partial \phi / \partial s_{b}, \quad \phi_{c}=\partial \phi / \partial s_{c}
$$

parallel to the element sides, the local freedoms are shown in Figure 1 (b).

In order to express the interpolation consistently in terms of dimensionless interpolation functions the natural derivatives are expressed as dimensionless derivatives,

$$
\phi_{a}^{*}=L_{a}\left(\partial \phi / \partial s_{a}\right)=x_{21} \phi_{x}+y_{21} \phi_{y} \quad \text { and similarly for } \phi_{b}^{*} \text { and } \phi_{c}^{*},
$$

where $x_{21}=x_{2}-x_{1}, y_{21}=y_{2}-y_{1}$ etc. and $s_{a}$ is a coordinate parallel to side 1-2. We note that $c_{a x}=x_{21} / L_{a}, c_{a y}=y_{21} / L_{a}$ are the direction cosines of side 1-2, $L_{a}$ being its length.

Including a centroidal freedom $\phi_{c}$ the complete cubic interpolation in areal coordinates is easily obtained as [8]

$$
\phi=\{f\}^{\prime}\left\{\phi_{1}, \phi_{2}, \phi_{3}, \phi_{a 1}^{*}, \phi_{c 1}^{*}, \phi_{b 2}^{*}, \phi_{a 2}^{*}, \phi_{c 3}^{*}, \phi_{b 3}^{*}, \phi_{c}\right\}=\{f\}^{\prime}\{d\},
$$

where

$$
\begin{aligned}
f_{i} & =L_{i}+L_{i}^{2}\left(\sum_{j} L_{j}\right)-L_{i}\left(\sum_{j} L_{j}^{2}\right)-9 L_{1} L_{2} L_{3} \quad(i=1,2,3) \quad\left(\text { for } \phi_{i}\right), \\
f_{2 i+2} & =L_{i}^{2} L_{i+1}-L_{1} L_{2} L_{3} \quad(i=1,2,3) \quad\left(\text { where } L_{4}:=L_{1}\right), \\
f_{2 i+3} & =-L_{i}^{2} L_{i+2}+L_{1} L_{2} L_{3} \quad(i=1,2,3), \\
f_{10} & =27 L_{1} L_{2} L_{3} \quad\left(\text { for } \phi_{c}\right)
\end{aligned}
$$

and the areal coordinates are given by cyclic permutation of

$$
L_{1}=1 / 3-\left(y_{32} x-x_{32} y\right) / 2 \Delta,
$$

where $2 \Delta=\left|x_{21} y_{32}-x_{32} y_{21}\right|$ gives the element area $\Delta$.

To eliminate the inconvenient centroidal freedom we can use the simple approximation [10]

$$
\phi_{c}=\left(\phi_{1}+\phi_{2}+\phi_{3}\right) / 3+\left(\phi_{a 1}^{*}-\phi_{c 1}^{*}+\phi_{b 2}^{*}-\phi_{a 2}^{*}+\phi_{c 3}^{*}-\phi_{b 3}^{*}\right) / 18
$$

Substituting (2.4) into the interpolation of (2.3), that is, into

$$
\phi=\sum_{i=1}^{9} f_{i} d_{i}+27 L_{1} L_{2} L_{3} \phi_{c}
$$

the modified interpolation functions are immediately obtained as

$$
\begin{aligned}
f_{i}^{*} & =f_{i}+9 L_{1} L_{2} L_{3} & & (i=1,2,3), \\
f_{2 i+2}^{*} & =f_{2 i+2}+(3 / 2) L_{1} L_{2} L_{3} & & (i=1,2,3), \\
f_{2 i+3}^{*} & =f_{2 i+3}-(3 / 2) L_{1} L_{2} L_{3} & & (i=1,2,3)
\end{aligned}
$$


and these are the interpolation functions of the classical BCIZ element [3], the simple explicit interpolation of which is still attractive.

Using (2.5), an interpolation matrix for the first derivatives is easily obtained as

$$
\left\{\partial() / \partial L_{1}, \partial() / \partial L_{2}, \partial() / \partial L_{3}\right\}=B\left(d^{*}\right\}
$$

where

$$
B^{\prime}=\left[\begin{array}{ccc}
1+L_{1}\left(2-L_{1}\right)-S & L_{1}^{2}-2 L_{2} L_{1} & L_{1}^{2}-2 L_{3} L_{1} \\
L_{2}^{2}-2 L_{1} L_{2} & 1+L_{2}\left(2-L_{2}\right)-S & L_{2}^{2}-2 L_{3} L_{2} \\
L_{3}^{2}-2 L_{1} L_{3} & L_{3}^{2}-2 L_{2} L_{3} & 1+L_{3}\left(2-L_{3}\right)-S \\
2 L_{1} L_{2}+a & L_{1}^{2}+b & c \\
-2 L_{1} L_{3}-a & -b & -L_{1}^{2}-c \\
a & 2 L_{2} L_{3}+b & L_{2}^{2}+c \\
-L_{2}^{2}-a & -2 L_{2} L_{1}-b & -c \\
L_{3}^{2}+a & b & 2 L_{3} L_{1}+c \\
-a & -L_{3}^{2}-b & -2 L_{3} L_{2}-c
\end{array}\right]
$$

and $S=L_{1}^{2}+L_{2}^{2}+L_{3}^{2}, a=L_{2} L_{3} / 2, b=L_{3} L_{1} / 2, c=L_{1} L_{2}$.

This relates to the local freedoms. Generalizing (2.2), the transformation from the global freedoms to these is simply

$$
\begin{aligned}
\left\{d^{*}\right\} & =\left\{\phi_{1}, \phi_{2}, \phi_{3}, \phi_{a 1}^{*}, \phi_{c 1}^{*}, \phi_{b 2}^{*}, \phi_{a 2}^{*}, \phi_{c 3}^{*}, \phi_{b 3}^{*}\right\} \\
& =T\{d\}=T\left\{\phi_{1}, \phi_{x 1}, \phi_{y 1}, \phi_{2}, \phi_{x 2}, \phi_{y 2}, \phi_{3}, \phi_{x 3}, \phi_{y 3}\right\}
\end{aligned}
$$

where

$$
T=\left[\begin{array}{ccccccccc}
1 & 0 & 0 & 0 & 0 & 0 & 0 & 0 & 0 \\
0 & 0 & 0 & 1 & 0 & 0 & 0 & 0 & 0 \\
0 & 0 & 0 & 0 & 0 & 0 & 1 & 0 & 0 \\
0 & x_{21} & y_{21} & 0 & 0 & 0 & 0 & 0 & 0 \\
0 & x_{13} & y_{13} & 0 & 0 & 0 & 0 & 0 & 0 \\
0 & 0 & 0 & 0 & x_{32} & y_{32} & 0 & 0 & 0 \\
0 & 0 & 0 & 0 & x_{21} & y_{21} & 0 & 0 & 0 \\
0 & 0 & 0 & 0 & 0 & 0 & 0 & x_{13} & y_{13} \\
0 & 0 & 0 & 0 & 0 & 0 & 0 & x_{32} & y_{32}
\end{array}\right]
$$

The derivatives of (2.6) are transformed to Cartesian derivatives

$$
\left\{\begin{array}{l}
\partial() / \partial x \\
\partial() / \partial y
\end{array}\right\}=(1 / 2 \Delta)\left[\begin{array}{ccc}
-y_{32} & -y_{13} & -y_{21} \\
x_{32} & x_{13} & x_{21}
\end{array}\right]\left\{\begin{array}{l}
\partial() / \partial L_{1} \\
\partial() / \partial L_{2} \\
\partial() / \partial L_{3}
\end{array}\right\}
$$




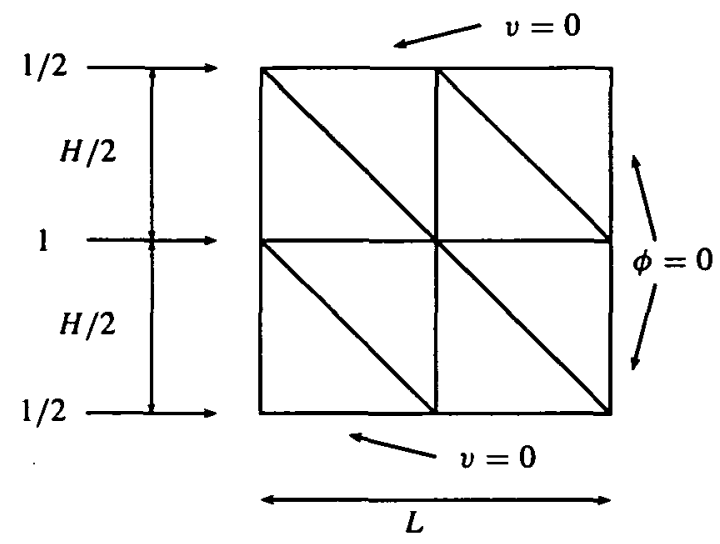

FIGURE 2. Rectilinear flow problem.

using the areal coordinate definitions given earlier, and the $2 \times 3$ matrix in (2.10) we denote by $Z$.

Then the final element matrix for Laplacian problems is given by a 6-point areal coordinate numerical integration of quartic accuracy ([8]) $k=\sum F F^{\prime}\left(\omega_{i} \Delta\right)$ with integration point weights $\omega_{i}$. The interpolation matrix $F$ for the two Cartesian derivatives is given by

$$
F=Z B T
$$

using (2.7), (2.8) and (2.10).

Finally, for the case of Poisson's equation, the 'local' consistent loads are given by the explicit integration [8] of (2.5), yielding

$$
\left\{q_{c}^{*}\right\}=(C \Delta) T^{t}\{8,8,8,1,-1,1,-1,1,-1\} / 24,
$$

where in the case of plane torsion $C=2 G \theta$. Using the simple matrix $T$ of (2.8) we obtain

$$
\begin{array}{cc}
q_{c 1}=q_{c 4}=q_{c 7} & =q \Delta / 3, \\
q_{c 2}=q \Delta\left(x_{21}-x_{13}\right) / 24, & q_{c 3}=q \Delta\left(y_{21}-y_{13}\right) / 24, \\
q_{c 5}=q \Delta\left(x_{32}-x_{21}\right) / 24, & q_{c 6}=q \Delta\left(y_{32}-y_{21}\right) / 24, \\
q_{c 8}=q \Delta\left(x_{13}-x_{32}\right) / 24, & q_{c 9}=q \Delta\left(y_{13}-y_{32}\right) / 24 .
\end{array}
$$

These explicit formulae are particularly useful and can be used for other $9 \mathrm{df}$ Hermitian triangular elements where simple formulae are not available [9].

The coding of the element is particularly simple and transformation by the matrix $T$ in (2.11) can, observing the simple form of (2.9), be coded explicitly rather than as a matrix multiplication. 


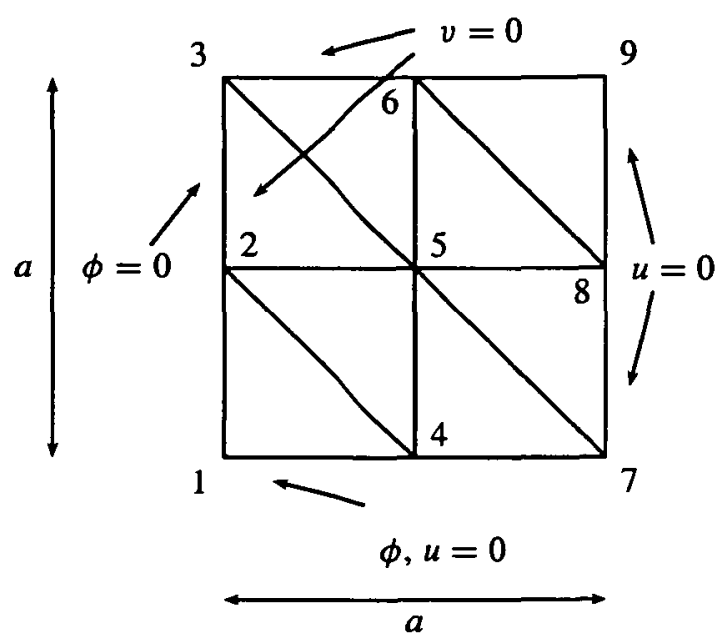

Figure 3. Plane torsion problem.

\section{A simple test problem}

The new element is first tested with the simple rectilinear flow problem shown in Figure 2 . Here $\phi$ is a potential function so that the flow velocities are given by

$$
u=-\partial \phi / \partial x, \quad v=-\partial \phi / \partial y .
$$

Equations (3.1) satisfy the irrotationality condition $\partial u / \partial y-\partial v / \partial x=0$. Substituting (3.1) into the continuity condition $\partial u / \partial x+\partial v / \partial y=0$ provides the governing PDE $\nabla^{2} \phi=0$.

To force the flow loads $q_{\phi}=1 / 2,1,1 / 2$ are specified at the inlet and $\phi=0$ is set as a datum at the outlet. It is also necessary to set $v=-\partial \phi / \partial y=0$ at the top and bottom of the domain.

This yields the expected results $u=-\partial \phi / \partial x=1, \phi_{\text {in }}=Q L / H$, where $Q=\sum q_{\phi}$ at the inlet.

\section{Convergence of the element}

The plane torsion problem is useful to test the convergence of the element. Here $\phi$ is a stress function such that $\tau_{z x}=\partial \phi / \partial y=-v, \tau_{z y}=\partial \phi / \partial x=u$ and the governing $\mathrm{PDE}$ is

$$
\nabla^{2} \phi+2 G \theta=0
$$


TABLE 1. Plane torsion solutions ( $\mathrm{LL}=$ lumped loads, $\mathrm{CL}=$ consistent loads).

\begin{tabular}{lcccc}
\hline Solution & Mesh 1 & Mesh 2 & Extrapolated & Basis \\
\hline $6 \mathrm{df}, \phi^{*}$ & 0.6000 & 0.5900 & 0.5893 & $h^{4}$ \\
$6 \mathrm{df}, \tau^{*}$ & 0.6500 & 0.6568 & 0.6590 & $h^{2}$ \\
$9 \mathrm{df}, \mathrm{LL}, \phi^{*}$ & 0.5357 & 0.5762 & 0.5897 & $h^{2}$ \\
$9 \mathrm{df}, \mathrm{LL}, \tau^{*}$ & 0.2143 & 0.3016 & $\mathrm{n} / \mathrm{a}$ & $\mathrm{n} / \mathrm{a}$ \\
$9 \mathrm{df}, \mathrm{CL}, \phi^{*}$ & 0.5714 & 0.5888 & 0.5894 & $h^{5}$ \\
$9 \mathrm{df}, \mathrm{CL}, \tau^{*}$ & 0.6786 & 0.6705 & 0.6702 & $h^{5}$ \\
Exact, $\phi^{*}$ & & & 0.5894 & \\
\multicolumn{1}{c}{$\tau^{*}$} & & & 0.6753 & \\
\hline
\end{tabular}

Figure 3 shows a quadrant of a square shaft section in plane torsion modelled using eight elements. The boundary conditions for the edges of the domain are shown, those at the corners combining those of the intersecting edges. Corresponding to the constant term of (4.1), 'lumped' loads of $q_{\phi 5}=2 a^{2} / 4, q_{\phi 6}=q_{\phi 8}=(1 / 2)\left(2 a^{2} / 4\right)$, $q_{\phi 9}=(1 / 4)\left(2 a^{2} / 4\right)$ are applied, in which $G \theta=1$ is assumed.

The results for $\phi$ at the centre $\left(\phi^{*}=\phi_{\max } / a^{2}\right)$ and $\tau$ at the middle of the sides $\left(\tau^{*}=\tau_{\max } / a^{2}\right)$ are compared to those of the 6-node quadratic Lagrangian element in Table 1. Here meshes with 9 nodes and df (mesh 1 ) and 25 nodes and $\mathrm{df}$ (mesh 2) are used for the $6 \mathrm{df}$ element and meshes with 4 nodes and $12 \mathrm{df}$ (mesh 1) and 9 nodes and $27 \mathrm{df}$ (mesh 2) are used for the new $9 \mathrm{df}$ element.

The results are extrapolated using $h^{N}$ extrapolation [8] and compared to those of the series solution [16]. For $\phi^{*}$ we require $N=2(p-m+1)=4$ and for $\tau^{*}$ we require $N=2$ for the quadratic element $[8,12]$.

For the cubic element, $N=2$ appears appropriate for $\phi^{*}$ but the results for $\tau^{*}$ are poor and cannot be extrapolated. The remedy is to use consistent loads (applying (2.12)), when $h^{5}$ extrapolation is found appropriate for both because the latter is now a nodal freedom.

This is intermediate between $N=4$ and $N=6$ for quadratic and cubic elements, not uncommon for elements involving approximations such as (2.4), and the results are clearly satisfactory.

Note that greater accuracy of stress or velocity solutions (with $N=5$ ) is a principal advantage of the cubic element, one that is demonstrated in the following section.

\section{Example}

Figure 4 shows the element applied to the classical problem of flow around a cylinder (one quadrant analysed) [7]. The loads $q_{\phi}$ shown are specified at the inlet and 


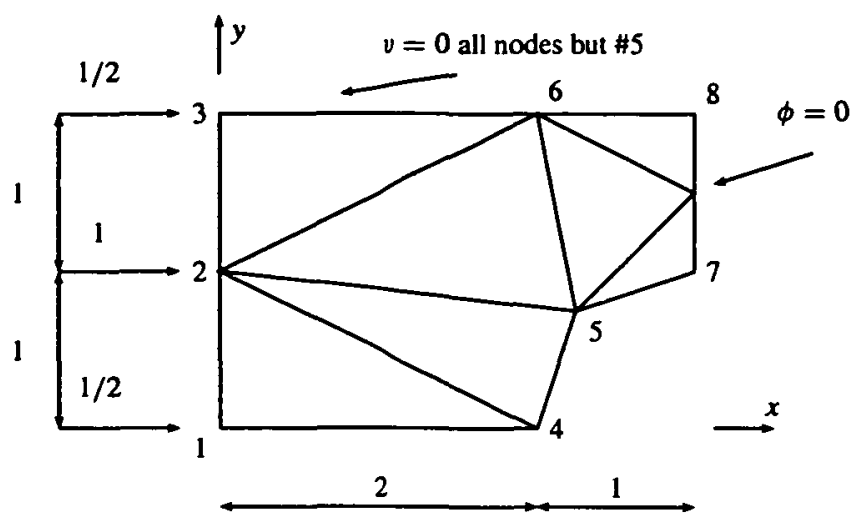

FIGURE 4. Flow around a cylinder.

TABLE 2. Results for the problem of Figure 4.

\begin{tabular}{cccc}
\hline Element & $u_{7}$ & $u_{8}$ & $u_{9}$ \\
\hline $3 \mathrm{df}$ & - & 1.232 & - \\
$6 \mathrm{df}$ & 1.756 & 1.930 & 1.826 \\
$9 \mathrm{df}$ & 2.506 & 1.834 & 1.757 \\
Exact & 2.509 & 1.884 & 1.755 \\
\hline
\end{tabular}

$\phi=0$ is set at the outlet, with $v=0$ around the boundary (except at node 5). The area 'cut out' by the cylinder is $A=\pi r^{2} / 4 \simeq 0.8$, so that the coordinates of node 5 are chosen as $x=2.2$ and $y=0.8$. This gives $A=1-2(1 / 2)(1)(0.2)=0.8$, yielding a reasonable approximation of the boundary shape.

The results for the velocity over the crest of the cylinder are compared in Table 2 to those obtained with the $3 \mathrm{df}$ linear element (using 10 nodes) [5], the $6 \mathrm{df}$ quadratic element (using 25 nodes) and exact solution [5].

The cubic element models the velocity profile above the crest of the cylinder well because the velocities are nodal freedoms, not the results of approximate 'stress type' calculations. The solutions for $\phi$ at the inlet are $3.93-4.00$, of the expected magnitude.

Therefore the simple cubic formulation is useful for accurate modelling of potential flow and other 'potential-type' problems.

\section{Infinite-boundary modelling}

Figure 5 shows a quadrant of a circular domain (of radius 4 ) with a point source at its centre modelled using (a) 6-node isoparametric elements [8] and (b) the present 


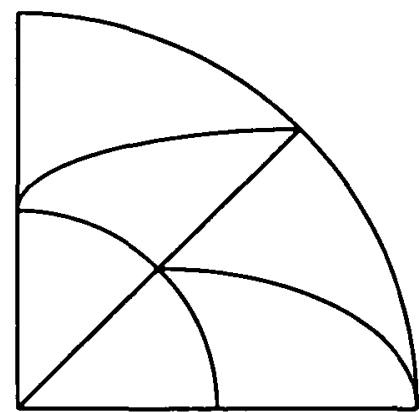

(a) 19 nodes

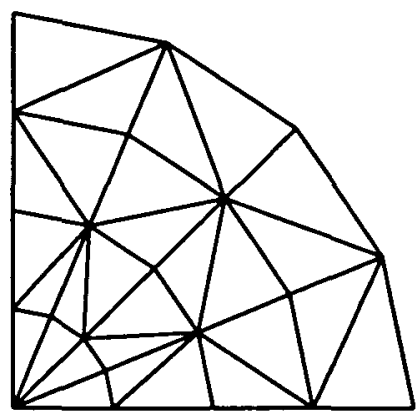

(b) 21 nodes

FIGURE 5. Two models of a quadrant of an infinite domain with a point source at the centre.

TABLE 3. Results for the problem of Figure 5.

\begin{tabular}{lcccc}
\hline Case & $r=1$ & $r=2$ & $r=3$ & $r=4$ \\
\hline 6 node FE (1a) & 525 & 367 & 276 & 210 \\
6 node FE (1b) & 520 & 365 & 275 & 209 \\
Cubic FE (2a) & 620 & 447 & 341 & 261 \\
Cubic FE (2b) & 520 & 374 & 284 & 218 \\
Expected (3) & 520 & 410 & 345 & 299 \\
Expected (4) & 480 & 370 & 305 & 259 \\
\hline
\end{tabular}

cubic element.

To simulate an infinite domain no boundary conditions are imposed other than $\phi=1000$ at the centre. However, in the fashion of the elastic boundary conditions used by Mohr and Power [14] for plane thermoelasticity problems, 'stiffnesses' equal to the angle (in radians) subtended by each node's 'share' of the boundary are added to the pivot for each boundary node's $\phi$ freedom before final solution of the problem. In Figure 5 (a), for example, the added values (in degrees) are 11.25 for the two nodes at $x=0$ and $y=0$ and 22.5 at the other three nodes of the circular boundary.

Table 3 shows the results obtained. The expected results are obtained by fitting the appropriate decay function which is $[6,17]$

$$
\phi=-(1 / 2 \pi) \phi_{0} \ln (r)+C .
$$

This is much used in the boundary element method [4].

In case (3) (row 5) the constant $C$ is calculated by substituting $\phi=520$ at $r=1$ in (6.1) and using the result to calculate $\phi$ for the other radii. Then for case (4) $\phi=370$ and $r=2$ is used to obtain row 6 .

Cases (1a) and (2a) are the FEM results with only conditions at infinity on the 
boundary. Here a 'natural' decay rate occurs in the meshes used. However in general the desired rate of decay should be modelled by choosing an appropriate $C$ value and setting a corresponding $\phi$ value as a boundary condition at an inner radius.

This is done for cases (1b) and (2b), setting $\phi=520$ for the nodes at $r=1$. The agreement of the two FEM results is now good and agreement with the expected (logarithmic) decay results is reasonable with such coarse meshes and such a rapid decay rate.

Finally, note that from (6.1) it follows that $\partial \phi / \partial r=-\phi_{0} /(2 \pi r)$. This can be set as a boundary condition in Figure 5 (at $r=4$ ) but this gives little change in the results of Table 3 (for the cubic element).

\section{Conclusions}

(1) The simple formulation for a $9 \mathrm{df}$ cubic element for potential flow problems, complete with explicit formulae for body force loads, is useful and accurate.

(2) The element uses the interpolations of the classical BCIZ element for thin plates, these being found in the course of the present work to be exactly equivalent to those of the (apparently quite different) Mohr and Mohr element.

(3) In element formulation, it is found useful to define natural velocities parallel to the (triangular) element sides. It is hoped that with further application of this approach in fluid mechanics the Navier-Stokes equations can be applied to each side of an element and that the usual continuity condition would then not be needed. This is the subject of current work.

(4) The results of Table 1 show that the element converges more rapidly than the popular $6 \mathrm{df} /$ node element as expected, but only if consistent loads are used. Here the plane torsion problem proves useful, along with the explicit formulae given for consistent loads.

In Table 2 the cubic element is clearly more accurate, though here many more freedoms are used for it.

(5) Though only small problems are studied here, the BCIZ element has been widely used for very large problems in structural mechanics and thus should be reliable in large fluids problems. However, in the present work it is the introduction of natural velocities in (2.1) to fluids problems and the use of conditions at infinity which are of primary interest.

(6) The conditions at infinity used for infinite domains are particularly simple and useful for a wide variety of problems, for example those of dipoles with only $\phi= \pm \phi_{0}$ as boundary conditions within the domain.

It is concluded, therefore, that the present formulation, its explicit formulae for consistent loads (and other details such as the use of (2.4) to obtain the final interpola- 
tions), the definition of natural velocities of flow and the simple conditions at infinity for infinite domains should all prove useful.

\section{References}

[1] J. H. Argyris, "Triangular elements with linearly varying strain for the matrix displacement method", J. Roy. Aero. Soc. 69 (1965) 711-713.

[2] J. H. Argyris, "Three-dimensional anisotropic and inhomogeneous elastic media, matrix analysis for small and large displacements", Ingenieur Archiv. 31 (1968) 33-55.

[3] G. P. Bazeley, Y. K. Cheung, B. M. Irons and O. C. Zienkiewicz, "Triangular elements in plate bending. Conforming and nonconforming solutions", in Proc. 1st Conf. Matrix Methods in Structural Mechanics, (WP AFB, Ohio, 1965).

[4] C. A. Brebbia, The boundary element method for engineers, 2nd ed. (Pentech Press, Plymouth, 1980).

[5] T. J. Chung, Finite element analysis in fluid dynamics (MGraw-Hill, New York, 1979).

[6] H. Lamb, Hydrodynamics, 5th ed. (CUP, Cambridge, 1924).

[7] H. C. Martin, "Finite element analysis of fluid flows", in Proc. 2nd Conf. Matrix Methods in Structural Mechanics, (WP AFB, Ohio, 1968).

[8] G. A. Mohr, Finite elements for solids, fluids, and optimization (OUP, Oxford, 1992).

[9] G. A. Mohr, "An accurate thin plate finite element", Comp. Meth. Appl. Mech. Engrg 166 (1998) 341-348.

[10] G. A. Mohr, "On two equivalent thin plate finite elements", Commun. Numer. Methods Engng 14 (1998) 271-275.

[11] G. A. Mohr and D. A. Caffin, "Penalty factors, Lagrange multipliers and basis transformation in the finite element method", Civil Engng Trans. Instn. Engrs Australia CE27(2) (1985) 174-180.

[12] G. A. Mohr and I. C. Medland, "On convergence of displacement finite elements, with an application to singularity problems", Engrg Fract. Mech. 17 (1983) 481-491.

[13] G. A. Mohr and R. S. Mohr, "A new thin plate element by basis transformation", Comput. Struct. 22 (1986) 239-243.

[14] G. A. Mohr and A. S. Power, "Elastic boundary conditions for finite elements of infinite and semi-infinite media", Proc. Instn. Civil Engrs (London) 65 (1978) 675-684.

[15] C. Pozrikidis, Introduction to theoretical and computational fluid dynamics (OUP, New York, 1997).

[16] S. P. Timoshenko and J. N. Goodier, Theory of elasticity, 3rd ed. (McGraw-Hill, New York, 1951).

[17] S. W. Yuan, Foundations of fluid mechanics (Prentice-Hall, Englewood-Cliffs, NJ, 1967).

[18] O. C. Zienkiewicz, The finite element method, 3rd ed. (McGraw-Hill, London, 1977). 